\title{
Evidence for a percolative Mott insulator-metal transition in doped $\mathrm{Sr}_{2} \mathrm{IrO}_{4}$
}

\author{
Zhixiang Sun $\odot,{ }^{1,2, *}$ Jose M. Guevara, ${ }^{2}$ Steffen Sykora, ${ }^{2,3}$ Ekaterina M. Pärschke, ${ }^{4}$ Kaustuv Manna $\odot,{ }^{2,5}$ Andrey Maljuk, ${ }^{2}$ \\ Sabine Wurmehl, ${ }^{2,6}$ Jeroen van den Brink, ${ }^{2}$ Bernd Büchner, ${ }^{2,6,7}$ and Christian Hess ${ }^{2,7,8, \dagger}$ \\ ${ }^{1}$ Center for Joint Quantum Studies and Department of Physics, Tianjin University, 300072 Tianjin, China \\ ${ }^{2}$ Leibniz-Institute for Solid State and Materials Research, IFW-Dresden, 01069 Dresden, Germany \\ ${ }^{3}$ Institute for Theoretical Physics, TU Dresden, 01069 Dresden, Germany \\ ${ }^{4}$ Department of Physics, University of Alabama at Birmingham, Alabama 35294, USA \\ ${ }^{5}$ Max-Planck-Institute for Chemical Physics of Solids, 01187 Dresden, Germany \\ ${ }^{6}$ Institute for Solid State Physics, TU Dresden, 01069 Dresden, Germany \\ ${ }^{7}$ Center for Transport and Devices, TU Dresden, 01069 Dresden, Germany \\ ${ }^{8}$ Fakultät für Mathematik und Naturwissenschaften, Bergische Universität Wuppertal, 42097 Wuppertal, Germany
}

(Received 3 November 2020; revised 31 March 2021; accepted 2 April 2021; published 27 April 2021)

\begin{abstract}
Despite many efforts to rationalize the strongly correlated electronic ground states in doped Mott insulators, the nature of the doping-induced insulator-to-metal transition is still a subject under intensive investigation. Here, we probe the nanoscale electronic structure of the Mott insulator $\mathrm{Sr}_{2} \mathrm{IrO}_{4-\delta}$ with low-temperature scanning tunneling microscopy and find an enhanced local density of states (LDOS) inside the Mott gap at the location of individual defects which we interpret as defects at apical oxygen sites. A chiral behavior in the topography for those defects has been observed. We also visualize the local enhanced conductance arising from the overlapping of defect states which induces finite LDOS inside of the Mott gap. By combining these findings with the typical spatial extension of isolated defects of about $2 \mathrm{~nm}$, our results indicate that the insulator-to-metal transition in $\mathrm{Sr}_{2} \mathrm{IrO}_{4-\delta}$ could be percolative in nature.
\end{abstract}

DOI: 10.1103/PhysRevResearch.3.023075

\section{INTRODUCTION}

Introducing defects in a Mott insulator leads to the fascinating appearance of different competing orders such as magnetism, superconductivity, or strange metallic behavior which includes enigmatic collective ordering states with a characteristic pseudogap. Local probes as for example scanning tunneling microscopy have successfully been used in the past to investigate these phenomena, particularly in the case of the well-established cuprate superconductors [1-3]. Recently, a new pathway to studying Mott physics was found by the discovery that the spin-orbit coupling driven pseudospin $J_{\text {eff }}=1 / 2$ Mott states of iridium oxide materials show an intriguing parallel to the spin $S=1 / 2$ Mott phases of the cuprate materials [4-6].

A prominent example is $\mathrm{Sr}_{2} \mathrm{IrO}_{4}$, which is a layered MottHubbard insulator with a weak antiferromagnetic order below $T_{\mathrm{N}} \simeq 240 \mathrm{~K}$ [7], where the magnetic moments are canted [8]. The material shares structural and magnetic similarities with the parent compounds of superconducting layered cuprates

\footnotetext{
*zsun@tju.edu.cn

$\dagger$ c.hess@uni-wuppertal.de
}

Published by the American Physical Society under the terms of the Creative Commons Attribution 4.0 International license. Further distribution of this work must maintain attribution to the author(s) and the published article's title, journal citation, and DOI.
[9]. Based on these observations, it has been argued that proper doping could drive the material into a high-temperature superconducting phase [10]. Indeed, recent experiments have shown that with electron doping a pseudogap feature and an arclike Fermi surface structure can be observed $[6,11,12]$. Furthermore, signatures of the spin-polaron physics well known from underdoped $\mathrm{La}_{2} \mathrm{CuO}_{4}$ and typical for the presence of strong electron correlations [13] were recently found in $\mathrm{Sr}_{2} \mathrm{IrO}_{4}$ using tunneling spectroscopy [14].

One possible method to drive the Mott insulating system $\mathrm{Sr}_{2} \mathrm{IrO}_{4}$ into a metallic state is to substitute the elements $\mathrm{Ir}$ or Sr through chemical doping [15-17]. Recently, the possibility to introduce electrons by substituting $\mathrm{La}$ for $\mathrm{Sr}$ has gained particular interest because the insulator-metal transition caused by this doping scheme has been reported to be connected with the formation of a pseudogap, reminiscent of the pseudogap phase of the cuprates [16,17], including a short-range stripelike charge ordering pattern trapped by the La atoms observed by scanning tunneling microscopy [12]. However, in all the above studies the mechanism of the enhanced conductivity by doping remains unclear.

A further way to turn $\mathrm{Sr}_{2} \mathrm{IrO}_{4}$ into a metallic phase is to introduce dilute oxygen vacancies into $\mathrm{Sr}_{2} \mathrm{IrO}_{4-\delta}$ where transport probes have found an insulator-metal transition at $\delta \approx 0.04$ [18]. Similar to the effect of La doping, the removal of oxygen is also expected to result in electron doping. Such oxygen depletion, as any other chemical doping scheme, also introduces disorder, which is expected to lead to the localization of states around the defects which may become 
particularly strong in a quasi-two-dimensional (2D) system [19]. Despite the expected localization caused by the impurities, it has been speculated, based on a variable range hopping analysis of resistivity data, that charge transport in oxygendeficient $\mathrm{Sr}_{2} \mathrm{IrO}_{4}$ is of percolative origin, where metallic patches are formed around the oxygen-depleted sites [18]. But an experimental proof is lacking and thus the underlying microscopic mechanism for the emergence of metallicity remains to be clarified.

Here, we investigate intrinsic defects in oxygen-deficient $\mathrm{Sr}_{2} \mathrm{IrO}_{4}$ using atomically resolved low-temperature scanning tunneling microscopy/spectroscopy (STM/STS) and probe the local electronic structure with a focus on the most probable apical oxygen site defects. We observe characteristic bound states inside the Mott gap in a confined area around isolated defects, which are naturally explained by impurity scattering of the correlated electrons of the Mott state. Interestingly, in regions with a spatial overlap of several defect areas a partial closing of the gap occurs. Based on this result we conclude that the insulator-metal transition in oxygen-deficient $\mathrm{Sr}_{2} \mathrm{IrO}_{4}$ arises from the percolative creation of conductive paths within an antiferromagnetic background. This finding suggests that the metallic state of the electron-doped $\mathrm{Sr}_{2} \mathrm{IrO}_{4}$ might be different from the strange metallic phase of weakly hole-doped cuprates where the common understanding is that the metallicity emerges upon the destruction of long-range order at low hole concentrations [20].

\section{EXPERIMENTAL DETAILS}

$\mathrm{Sr}_{2} \mathrm{IrO}_{4-\delta}$ single crystals with reduced resistivity due to oxygen deficiency were grown by the flux method [21]. The STM measurements were performed in a home-built variable temperature STM [22], using a PtIr tip. The single crystals were cleaved inside the STM head in cryogenic vacuum at about $9.0 \mathrm{~K}$ with the $c$ axis as the surface normal. All the STM measurements are carried out also at a temperature of $9.0 \mathrm{~K}$.

For the measurements of the tunneling conductance spectra we applied the standard lock-in method with an external modulation of $1.1111 \mathrm{kHz}$. The root mean square of the alternating voltage modulation that we applied for the acquisition of the tunneling spectra is $20 \mathrm{mV}$.

\section{RESULTS}

Figures 1(a) and 1(b) show a $14 \mathrm{~nm} \times 14 \mathrm{~nm}$ field of view of the atomically resolved topography data of a freshly cleaved sample surface, measured at $T=9 \mathrm{~K}$ using bias voltages $U=1.0 \mathrm{~V}$ and $U=-0.4 \mathrm{~V}$, respectively. Since the natural cleaving plane of $\mathrm{Sr}_{2} \mathrm{IrO}_{4}$ is in between the $\mathrm{SrO}$ double layers, we attribute the visible corrugation to either the $\mathrm{Sr}$ or to the apical oxygen ions. Interestingly, the amplitude plot of the Fourier transform [Fig. 1(d)] reveals not only the Bragg peaks of a 2D square lattice but also a $(1 \times 1)$ superstructure. It is consistent with a planar symmetry reduction which arises from an in-plane rotation of the $\mathrm{IrO}_{6}$ octahedra [see Fig. 1(c)] by about $12^{\circ}$ along the $c$ axis [23].

Let us first characterize the visible defects in the topographic data. A large-scale count (see Appendix A) yields a total abundance of about $2.6 \%$ per Ir atom. The defects can

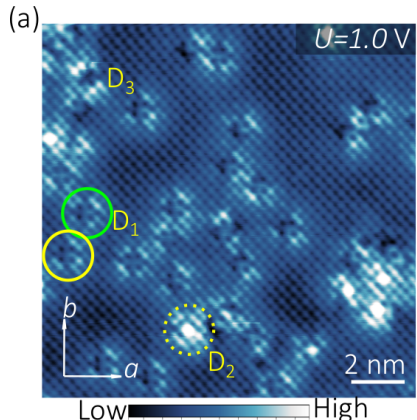

(c)

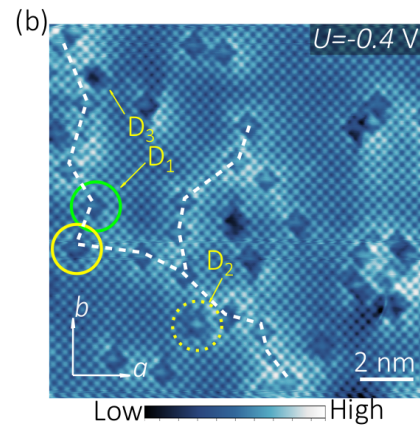

(d)
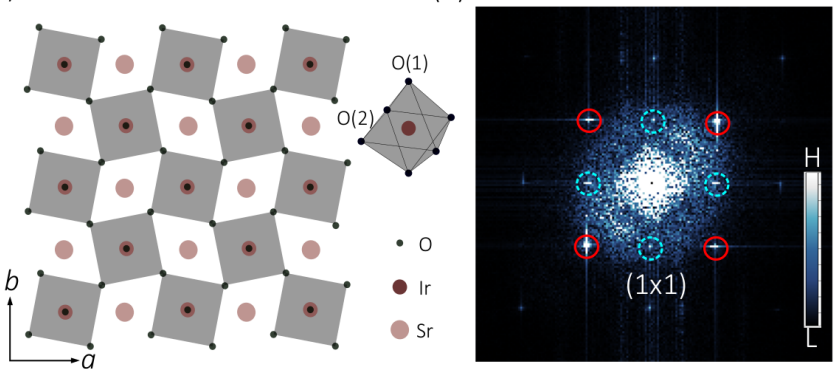

FIG. 1. Surface topography and the different types of defects. (a), (b) Topography of the sample surface measured at $U=1.0 \mathrm{~V}$ and $U=-0.4 \mathrm{~V}$, respectively. Characteristic defects are marked by circles. The solid circles mark out the two $\mathrm{D}_{1}$ defects with different chirality with green (upper) and yellow (lower) color, and the dashed one marks the $\mathrm{D}_{2}$ defect. The white dashed lines indicate possible paths of percolative charge transport. (c) A top view of the lattice structure in the $a b$ plane of $\mathrm{Sr}_{2} \mathrm{IrO}_{4}$. On the right-hand side octahedron, the vertical direction is along the $c$ axis. $\mathrm{O}(1)$ is the apical oxygen and $\mathrm{O}(2)$ is the basal oxygen. (d) The amplitude of the Fourier transform of the topography image (b), where we label the Bragg peaks with solid (red) circles and the peaks due to the $(1 \times 1)$ lattice distortion with dashed circles.

be differentiated by their distinct appearance at positive and negative bias voltages into three types denoted by $D_{1}, D_{2}$, and $\mathrm{D}_{3}$, as is illustrated in Figs. 1(a) and 1(b). The two types $\mathrm{D}_{1}$ and $\mathrm{D}_{2}$ are located at the same lattice site in the $a b$ plane but can be clearly distinguished from their height profiles in the topography taken at positive bias. The apparent height difference for the two types of defects is about $80 \mathrm{pm}$ (see Appendix A). Since our sample possesses a significantly reduced resistivity due to oxygen deficiency [14], it is reasonable to attribute this effect to the missing oxygen [18]. On this basis, we tentatively assign the visible regular atomic corrugation to the $\mathrm{Sr}$ atoms of the topmost layer, and the defects $\mathrm{D}_{1}$ and $\mathrm{D}_{2}$ to the apical oxygen $[\mathrm{O}(1)$ in Fig. 1(c)].

A more careful inspection of the topographic data reveals that the intensity distribution in the environment of $\mathrm{D}_{1}$ defects has a certain chirality depending on the bias voltage at which the topography is recorded. Thereby, the classification signature is given by all four nearest-neighbor atomic pairs being tilted in the same chiral direction. We observe that this chirality depends on the lattice site: By analyzing the topographies of individual defects, we find that the chirality behavior is different for defects on the nearest octahedra. This is a clear breaking of the translational symmetry. This feature, as the $(1 \times 1)$ superstructure, is naturally explained by the rotation 

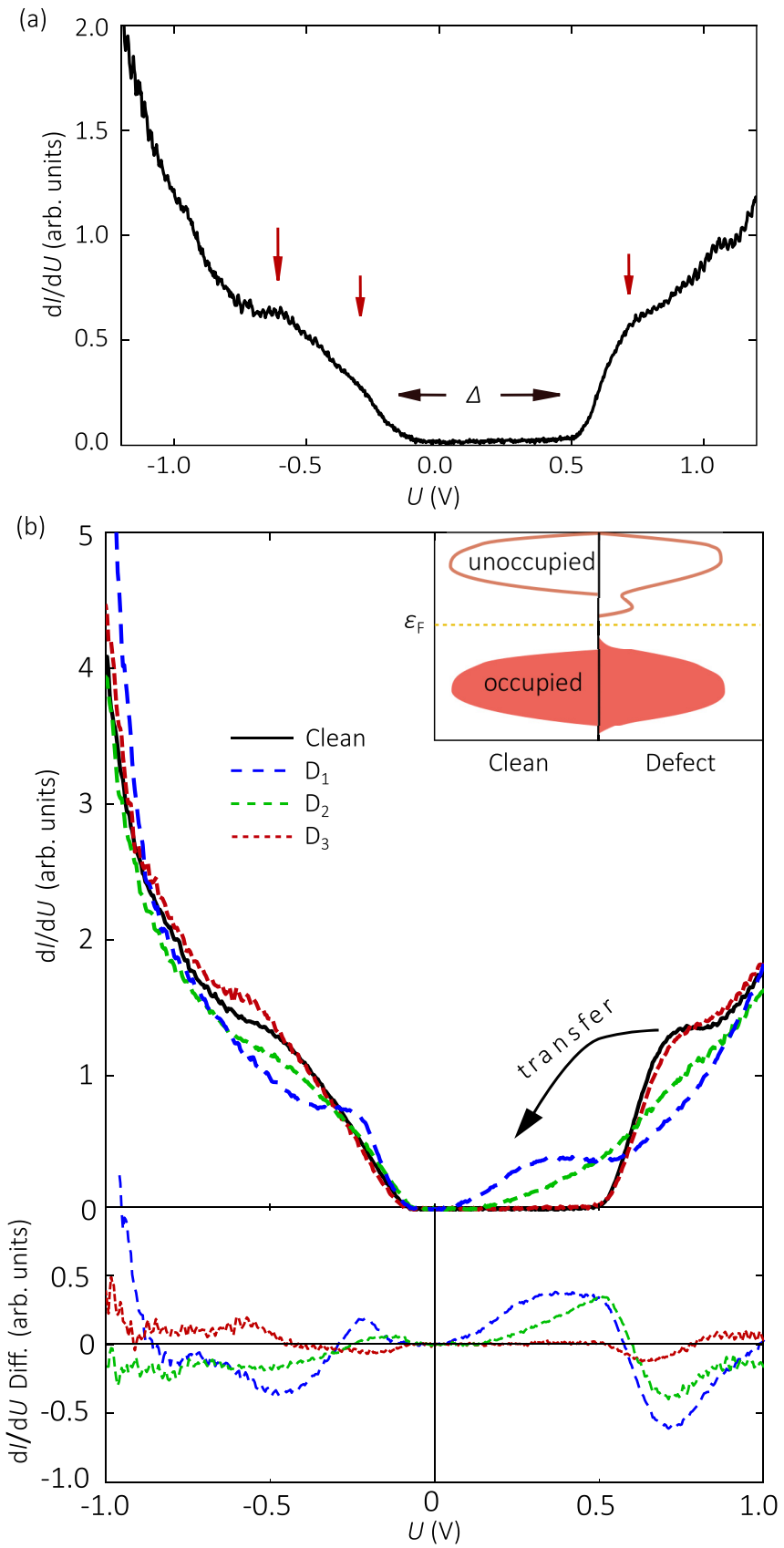

FIG. 2. Differential tunneling conductance spectra. (a) Overview tunneling conductance spectrum taken at a clean place. (b) Spectra taken on top of the $\mathrm{D}_{1}, \mathrm{D}_{2}$, and $\mathrm{D}_{3}$ defects as discussed in the text. Lower panel: The differences between the spectra taken on the clean places and on top of the defects. The inset illustrates the transfer of the spectral weight into the gap on top of the apical oxygen site defects.

of the $\mathrm{IrO}_{6}$ octahedra and is well known from previous STM measurements on the related double-layer perovskite $\mathrm{Sr}_{3} \mathrm{Ir}_{2} \mathrm{O}_{7}$ [24]. It seems worthwhile to note that the apparent chirality reverses as the bias voltage changes its sign. A more detailed description and analysis of the observed chirality can be found in the Appendix B.

A third defect type $\left(D_{3}\right)$ with a much lower surface abundance than $D_{1}$ and $D_{2}$ appears as a hole on the sample surface and is located at the surface site of the Sr ions [Fig. 1(b)]. Unlike $\mathrm{D}_{1}$ and $\mathrm{D}_{2}$, which, as we will show further below, have a profound impact on the local density of states (LDOS), the effect of $D_{3}$ is rather weak. This indicates for $D_{3}$ either the possibility of a missing $\mathrm{Sr}$ atom or an isovalent impurity ion such as the light earth alkalines $\mathrm{Ca}$ or $\mathrm{Mg}$.

From the topographic data in Figs. 1(a) and 1(b) we clearly see an enhanced intensity within a few lattice constants around the defects. Consequently, since the topography describes the energy-integrated LDOS we expect an appearance of new states near the Fermi level around the defects (bound states). These states may combine to conglomerates of conductive patches as indicated by the dashed lines in Fig. 1(b) and offer the possibility of percolative charge transport. To confirm this expectation we have measured the tunneling spectrum on top of the discussed defect types and in a clean area of the sample. The result is shown in Fig. 2. In the spectrum taken on a place free of defects [Fig. 2(a), the same as in Ref. [14]] we can clearly see the Mott gap $\Delta \approx 620 \mathrm{meV}$ which is consistent with results from previous STM measurements $[11,25]$. Outside this gap there are features (indicated by red arrows) which are the signature of spin-polaron physics [14]. The impact of the defects is shown in Fig. 2(b). While on the $D_{1}$ and $D_{2}$ defect types $\Delta$ is reduced to a value of less than $150 \mathrm{meV}$, there is no significant decrease for the $D_{3}$ type. For the $D_{1}$ defect at both polarities the spectral weight is clearly shifted towards the Fermi level, connected with peaklike features at about -200 and $250 \mathrm{meV}$, resulting in a remaining gap of about $100 \mathrm{meV}$. This transfer of spectral weight is seen more clearly in the difference of the spectra taken on defects and taken on the clean place as plotted in the lower panel of Fig. 2(b). While for $\mathrm{D}_{1}$ a clear resonance peak inside the gap is found, the filling of the gap with spectral weight is also present for $\mathrm{D}_{2}$ but is more smooth and less resonantlike.

\section{DISCUSSION}

The appearance of the in-gap states can be confirmed by considering theoretically the effect of on-site potential scattering caused by local defects/charge. For this we

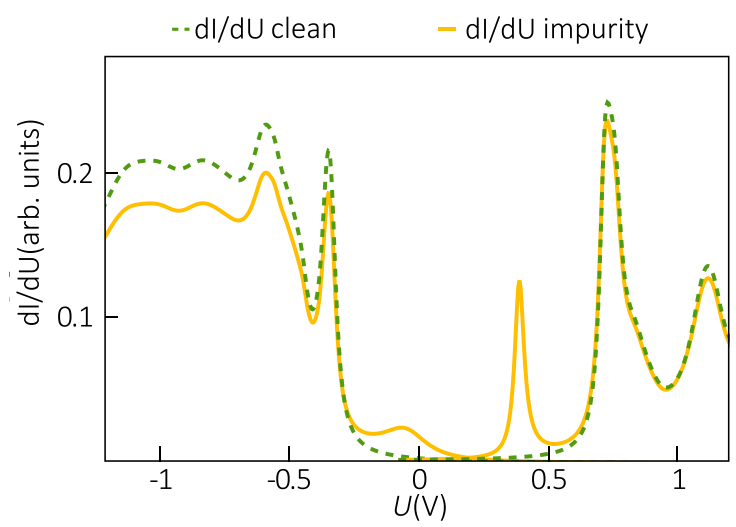

FIG. 3. Calculated effect of a local defect to the differential conductance. The spectrum (yellow line) is characterized by an on-site Coulomb scattering potential with a strength of $V_{\text {imp }}=0.028 \mathrm{eV}$. The corresponding differential conductance calculated for the clean system is also shown (green dashed line). 
(a)

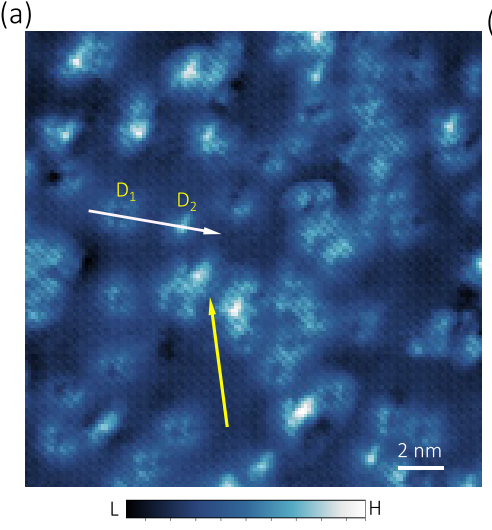

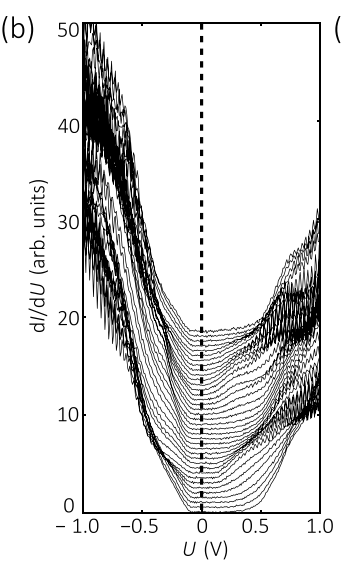

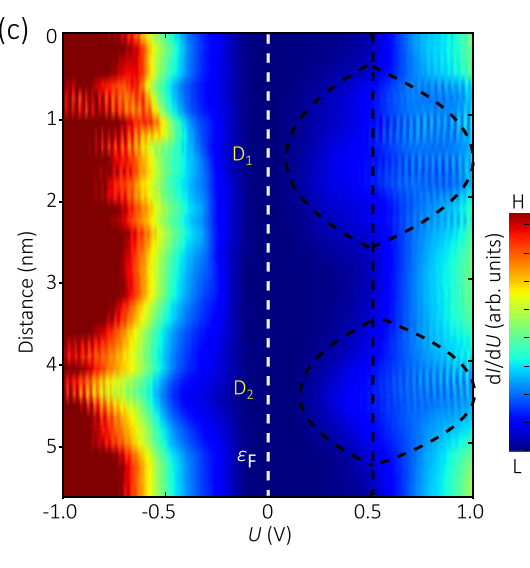

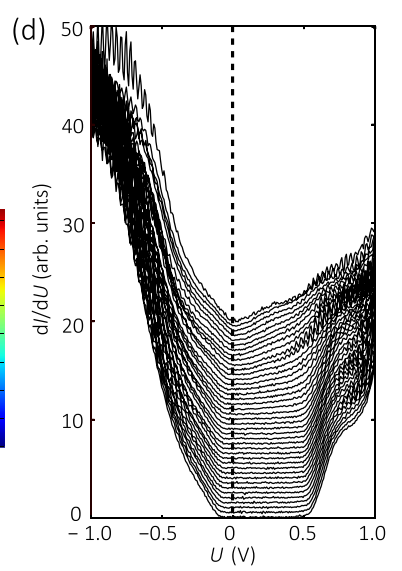

FIG. 4. The spatial electronic effects of different defects. (a) The corresponding topography where we have taken the spectroscopic mapping. Set point: $U=1.0 \mathrm{~V}, I=200 \mathrm{pA}$. (b) The line profile of the spectra along the white arrow as shown in (a) crossing the two different types of defects, $\mathrm{D}_{1}$ and $\mathrm{D}_{2}$, shown with a constant offset for clarity. (c) The corresponding pseudocolor spatial map of the spectral intensity profile shown in (b). (d) Line profile of the differential tunneling conductance spectra along the yellow arrow shown in (a).

employed the theoretical model describing spin polarons in $\mathrm{Sr}_{2} \mathrm{IrO}_{4}$, which we introduced in Ref. [14] to describe the spectral signatures of a clean system. Here, we additionally introduced a local defect and calculated its effect to the differential conductance on the basis of a $t$-matrix approach (see Appendix C 1). As shown in Fig. 3, characteristic bound states appear inside the Mott gap on top of a defect. As observed in our experiment, the defect produces spectral weight near the Fermi level which appears additionally to the spin-polaron excitations of the clean Mott system. If the distance of the individual defects is small enough these bound states combine and may form impurity bands which can contribute to charge transfer.

To underpin this picture, we experimentally investigate in Fig. 4 the spatial extension of the defect bound states and show the variation of the tunneling spectrum crossing defects of type $D_{1}$ and $D_{2}$. Thereby we study the evolution of the spectra along two different directions in the sample [indicated by white and yellow arrows in Fig. 4(a)]. In the first case (along the white arrow) the bound states of the affected defects are rather distinct. We can see that the effect is very localized at the defects and the Mott gap does not fully close on the individual defects [see Fig. 4(b)]. The spatial extension of the individual defect states is better seen in Fig. 4(c) where the line profile of the spectra is illustrated using a pseudocolor scale. For $\mathrm{D}_{1}$ and $\mathrm{D}_{2}$ the bound states appear in areas of about 2 and $1.5 \mathrm{~nm}$ in diameter, respectively. The absence of states at the Fermi level [white dashed line in Fig. 4(c)] clearly indicates that the ground state is still insulating, even on top of the defects.

In the second line profile [along the yellow arrow in Fig. 4(a)] where we track the spectra starting from a clean region of the sample into an area with a large defect density the behavior changes dramatically. Within the area of high defect density the gap nearly closes, which indicates that we have entered a region where charge transport is possible. This observation indicates the possibility that the entered region is a part of a path along which percolative charge transfer can happen.
To answer the question at which doping such percolative transport is possible we have performed a standard bond percolation simulation (see Appendix C 2) for a 2D square lattice where we have fixed the size of the bond to four lattice constants (which corresponds to a 2-nm extension of the impurity effect as found from Fig. 4). From this calculation we found a percolation threshold of $3.7 \%$ defect concentration over the Ir atoms. Interestingly, this value is remarkably close to the critical doping of $\delta \approx 0.04$ where the insulator-to-metal transition is found in single-crystal $\mathrm{Sr}_{2} \mathrm{IrO}_{4-\delta}$ [18]. This agreement of the percolation threshold with the critical doping level of the insulator-metal transition provides very strong evidence that the metallic state is realized by percolative conductive paths of overlapping impurity sites within an otherwise only weakly altered Mott insulating antiferromagnetic background. We stress that the metallic state has been reported to exhibit antiferromagnetic order in practically the same temperature range as pristine $\mathrm{Sr}_{2} \mathrm{IrO}_{4}[18,26]$, which supports our scenario. Based on these theoretical and experimental values for the percolation threshold, our sample with a measured defect density of $2.6 \%$ with respect to the Ir atoms should be located at the insulating side of the percolation threshold. Indeed, the bulk resistivity measurements of the sample reveal nonmetallic behavior with significantly reduced insulating properties (as compared to quasistoichiometric $\mathrm{Sr}_{2} \mathrm{IrO}_{4}$ ) as shown in Appendix D. Thus both the bulk transport, and the LDOS data of our sample are well consistent with each other. The reduced resistivity and the extended path fragments suggest that the sample electronic properties are close to the verge of metallicity.

We mention that a similar closing of the Mott gap induced by oxygen vacancies has been seen previously by probing the differential conductance for the closely related higher-order Ruddlesden-Popper iridate material $\mathrm{Sr}_{3} \mathrm{Ir}_{2} \mathrm{O}_{7}$, albeit for a significantly smaller Mott gap $(\sim 130 \mathrm{meV}$ for the clean material) [24]. The authors of this study argued that the origin of the gap filling could be a defect-induced reduction of the on-site Coulomb repulsion. According to band structure calculations this effect would cause an almost rigid shift of the respective 


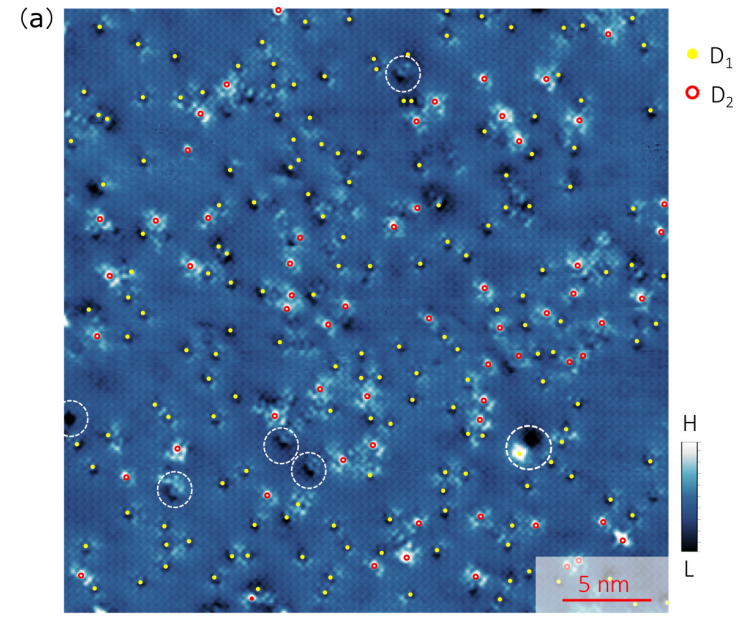

(b)
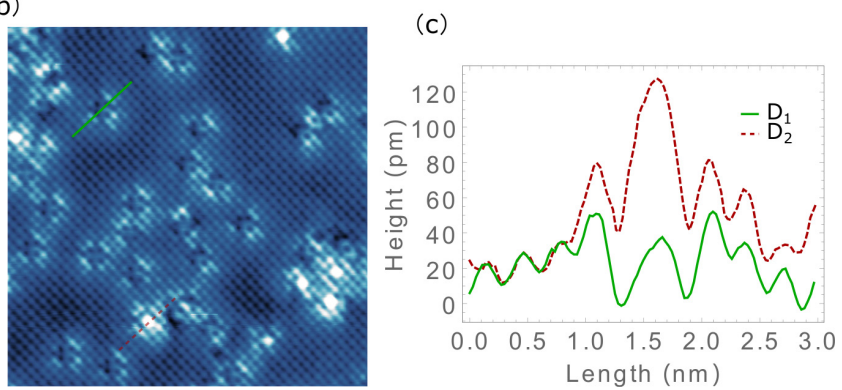

FIG. 5. (a) Surface defect density counting. A large area topography of the sample surface, taken with the parameter values $U=1.0$ $\mathrm{V}, I=200 \mathrm{pA}$. From the image we can see that the intrinsic defects are rather homogeneous. We have marked out two types of defects, $\mathrm{D}_{1}$ (yellow dot) and $\mathrm{D}_{2}$ (red circle). (b) The same topography as Fig. 1(a), with the two line profiles for two different defects. (c) The height line profiles of the two lines marked in (b).

two parts of density of states towards each other which are related to the lower and upper Hubbard band edges. Such a scenario is, however, incompatible with our data since the spectral weight which is transferred into the gap at a single impurity clearly appears as distinct peaks, characteristic of bound states, at both edges of the gap rather than a shift of the band edges [see Fig. 2(b)]. We therefore conclude that the new in-gap states indeed are the result of impurity scattering, as calculated in Fig. 3.

It is further interesting to note that the $d I / d U$ at high impurity density as shown in Fig. 4(d) strongly resembles that of the reported pseudogap in La-doped $\mathrm{Sr}_{2} \mathrm{IrO}_{4}$ [12]. Indeed, in such a doping scheme a similar scenario for the insulatormetal transition arising from overlapping impurity scattering bound states might apply. In this case the percolative conduction paths might be caused by the $\mathrm{La}^{3+}$-ion impurity potential. While a careful on-site defect spectroscopy, as we did here for the oxygen defects, should be able to unequivocally clarify such a notion. The importance of the impurity scattering strength for the formation of states inside the Mott gap is underpinned by a recent study of La-doped $\mathrm{Sr}_{3} \mathrm{Ir}_{2} \mathrm{O}_{7}$ [27]. Those results clearly show that the impact of the dopants on the electronic structure decisively depends on their lattice position and thus the strength of the impurity potential within the $\mathrm{IrO}_{2}$ layers.

\section{CONCLUSION}

To conclude, in this work we have investigated the effect of intrinsic defects of oxygen-deficient $\mathrm{Sr}_{2} \mathrm{IrO}_{4}$ which we assign to missing oxygen at the apical $\mathrm{IrO}_{6}$ octahedra. We have shown that characteristic bound states are formed within a spatial scale of around $2 \mathrm{~nm}$, creating spectral weight inside the Mott gap. For isolated defects the gap does not fully close, but when defects start to spatially overlap, the gap closing becomes almost complete. Therefore, our local probe indicates the scenario of filamentary metalliclike conductivity through agglomerations of defects. This interpretation is fundamentally different from the usual mechanism of the insulator-to-metal transition in the underdoped cuprates which usually is understood as to originate from the correlated motion of charge in an antiferromagnetic background. Our finding is corroborated by a simulation of the bond percolation threshold based on realistic parameters of the lateral extension of the impurity states, where we find a very good agreement with the experimental critical doping level.

\section{ACKNOWLEDGMENTS}

We thank C. Renner, B. J. Kim, V. Madvahan, and M. Allan for helpful discussions and D. Baumann and U. Nitzsche for technical assistance. The project is supported by the Deutsche Forschungsgemein-schaft through SFB 1143 (project-id 247310070), and by the Emmy Noether programme [S.W. Project No. WU595/3-3, S.S. Project No. ME4844/1-1 (project-id 327807255)]. Furthermore, this project has received funding from the European Research Council (ERC) under the European Unions' Horizon 2020 research and innovation programme (Grant Agreement No. 647276-MARS-ERC-2014-CoG).

\section{APPENDIX A: DEFECT IDENTIFICATION AND DEFECT DENSITY}

Since the STM measurement does not have the ability to identify the chemical elements, we cannot provide direct confirmation for the statement that the $D_{1}$ and $D_{2}$ defects are located at the apical oxygen sites. We therefore argue based on the lattice structure.

The data show that the exact locations of the $D_{1}$ and $D_{2}$ defects in the $a b$ plane are located in between the regular surface protrusions in the $\mathrm{SrO}$ surface. If we assign the peaks in the regular corrugation to the $S r$ atoms, then the $D_{1}$ and $D_{2}$ defects must be located at the apical oxygen sites. If, on the other hand, the peaks of the regular corrugation were located at the apical oxygen sites, then the $\mathrm{D}_{1}$ and $\mathrm{D}_{2}$ defect location would correspond to the lattice site of the $\mathrm{Sr}$ atom. Since $\mathrm{Sr}$ atoms have a weak contribution to electronic states at low energy, it is hard to understand the rather strong modulation to the local electronic structure. Therefore we conclude that the defect lattice site most likely corresponds to the position of apical oxygen. Since there are two types of defects on this lattice site we can further assign them to the two apical oxygens in the octahedron [see Fig. 1(c)] because it is clear that they should appear differently in the STM topography. Furthermore, such defects should occur in oxygen-deficient $\mathrm{Sr}_{2} \mathrm{IrO}_{4}$ (evidenced by the low resistivity of our sample, see Appendix D), as missing oxygen at the apical oxygen sites, 
(a)

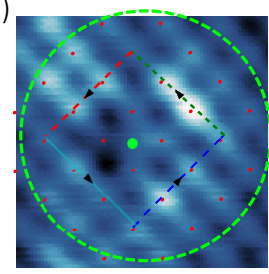

anti-CLW

(b)

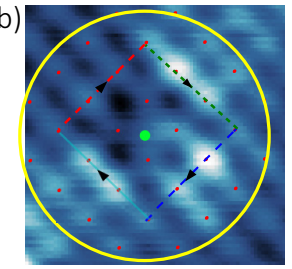

CLW

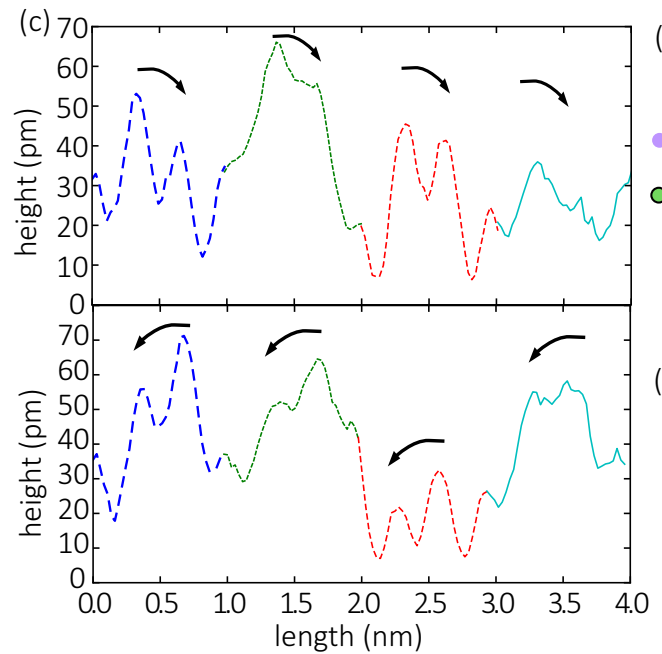

(d)

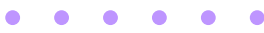

Sr

o Def

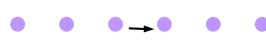

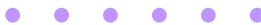

(e)
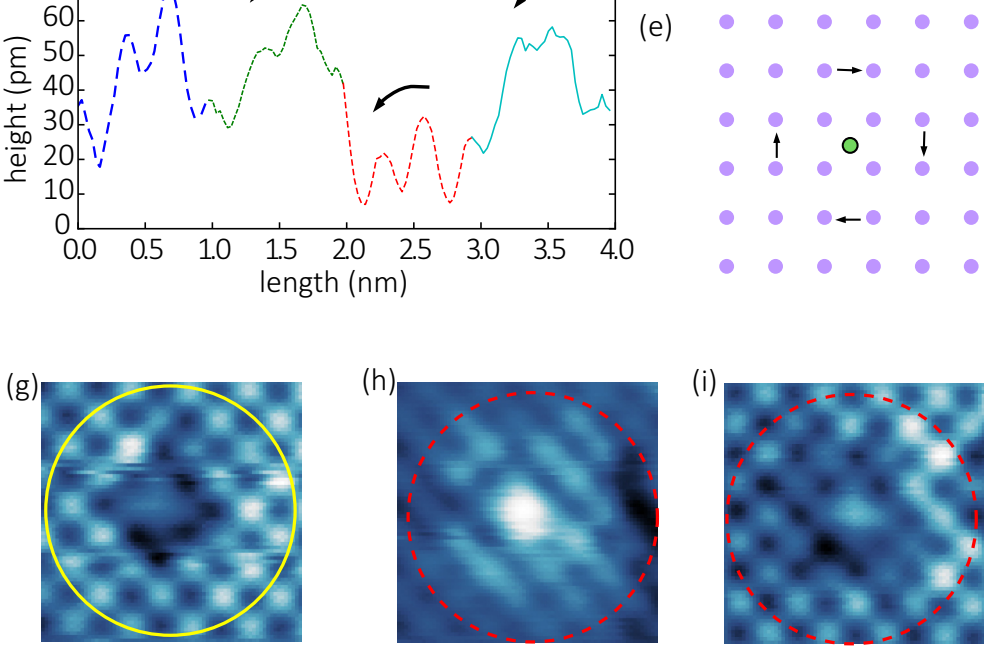

FIG. 6. Chiral behavior of the defect topographies and their bias dependencies. The defect topographies are taken from Fig. 1. (a), (b) The anticlockwise and clockwise behavior around the $\mathrm{D}_{1}$-type defects, which are taken from Fig. 1(a). The bias voltage is 1.0 eV. The red dots are the positions of the surface Sr atom. (c) The line profiles of the topography marked in (a) and (b), where the arrows are the guide to eyes on the change of the height profile. (d), (e) Illustrate the chirality behavior observed in the topography (a) and (b), respectively. (f), (g) The topographies of the two defects in (a) and (b) taken with a negative bias. (h) $\mathrm{A} \mathrm{D}_{2}$-type defect topography taken with a positive bias. (i) The $\mathrm{D}_{2}$-type defect [shown in (h)] topography taken with a negative bias.

consistent with our observation. Hence, the most reasonable location of the $\mathrm{D}_{1}$ and $\mathrm{D}_{2}$ defects is at the apical oxygen sites, where it is reasonable to attribute them to missing oxygen.

We noticed that the $\mathrm{D}_{1}$ and $\mathrm{D}_{2}$ defects have different surface densities. One of the large area topographies is shown in Fig. 5. We can see that the $\mathrm{D}_{1}$ defects have a larger surface density, about $2 \%$ over the Ir atoms. The surface density of the $\mathrm{D}_{2}$ defects is about $0.6 \%$ over the Ir atoms.

Besides the $\mathrm{D}_{1}, \mathrm{D}_{2}$, and $\mathrm{D}_{3}$ types of defects, we have also observed some rare defect types [labeled with dashed circles in Fig. 5(a)]. Those defects appear much less frequently as compared to the $\mathrm{D}_{1}, \mathrm{D}_{2}$, and $\mathrm{D}_{3}$ defects. Identifying their origin and probing their effects on the local electronic structure should be also an interesting subject in future studies.

\section{APPENDIX B: CHIRAL FEATURE OF DEFECTS}

A more careful comparison of the topographic appearance of the defects $D_{1}$ and $D_{2}$ reveals that each of these defect types possesses two variants which differ in the chirality of the surrounding topography. This can be inferred from the closeups of the topography of two different $D_{1}$ defects as shown in Figs. 6(a) and 6(b). While the four nearest surface atoms around the defect are hard to resolve, the respective height profiles of the next-nearest $\mathrm{Sr}$ atoms represent the signature of the different chirality quite clearly. This signature is emphasized in the respective height profiles of the next-nearest $\mathrm{Sr}$ atoms around the defects [Fig. 6(c)] and the illustrated chiralities [Fig. 6(d) and 6(e)]. This topographic chiral character is also visible at negative bias voltage as can be inferred from Fig. 6(f) and 6(g). Apparently, the chiral signature in the height profile is opposite from that identified at positive bias (see Fig. 6). We investigate the presence of the chiral dichotomy also for the $\mathrm{D}_{2}$ defects in Figs. 6(h) and 6(i) which show the same $\mathrm{D}_{2}$ defect at positive and negative bias voltage (see Fig. 7). However, although we cannot exclude its presence, a chiral pattern is difficult to discern and remains beyond the resolution.

Based on our assumption that the $D_{1}$ and $D_{2}$ defects are located at the apical oxygen sites, the chiral feature around these defects is naturally explained by the known rotation of the $\mathrm{IrO}_{6}$ octahedra. We mention that a chiral structure around the $\mathrm{D}_{1}$ defects has also been observed in previous STM measurements on the related double-layer perovskite $\mathrm{Sr}_{3} \mathrm{Ir}_{2} \mathrm{O}_{7}$ [24], which possesses a similar in-plane lattice distortion.

In order to demonstrate the intimate connection between the chirality and the lattice distortion, we investigate the distance dependence of the chirality between individual $D_{1}$ defects. If the observed chirality possesses the same lattice periodicity as that of the rotated octahedra in the plane, one would expect the chirality to be the same (different) for every even (odd) multiple Ir-Ir distance along the [110] or [110] direction [see Fig. 1(c)]. 
(a)

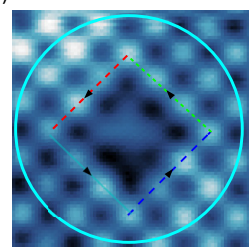

$\mathrm{D}_{1}$

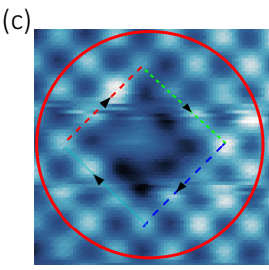

$D_{1}$

(e)

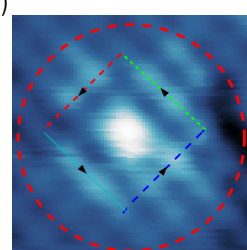

$\mathrm{D}_{2}$

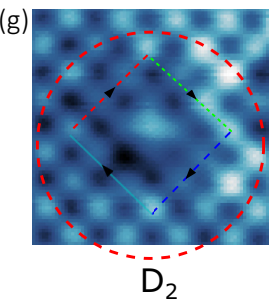

(b)

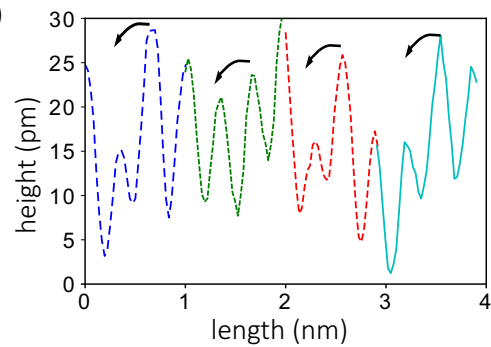

(d)

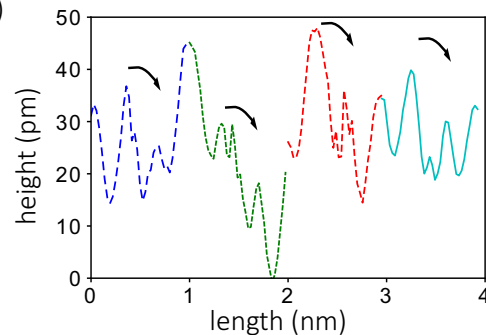

(f)

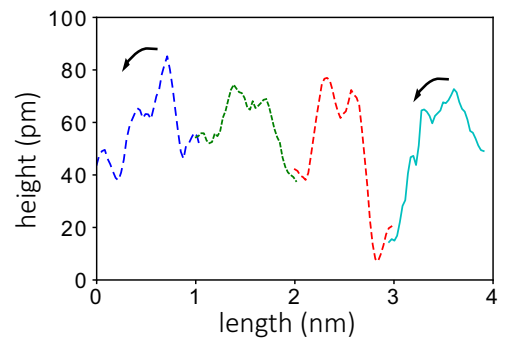

(h)

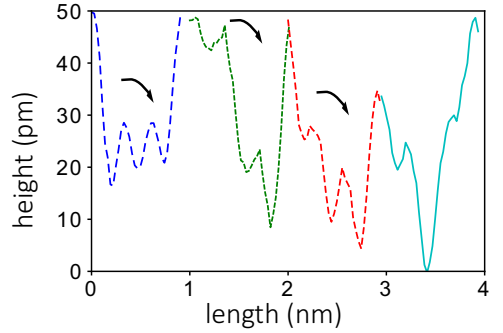

FIG. 7. More topographic height line profiles around $\mathrm{D}_{1}$ - and $\mathrm{D}_{2}$-type defects shown in Fig. 1. (a), (c), (e), (g) Topographies of individual defects. (b), (d), (f), (h) The corresponding line profiles around the defects.

Let us first define the distance $R$ between two defects as the steps needed to get from one to the other by moving along the nearest Ir-Ir lattice directions in units of $a_{0}$, where $a_{0}$ is the distance between the two nearest Ir atoms. Then the $\bmod \left(R, 2 a_{0}\right)$ should be either 0 or $a_{0}$, where mod is the modulo operation. According to our observation, defects obeying $\bmod \left(R, 2 a_{0}\right)=$ 0 have the same local chirality. In Fig. 8, we have marked out six $\mathrm{D}_{1}$-type defects as $\mathrm{D}_{1 i}(i=a, \ldots, f)$. Then we can apply the modulo computation to the distances between any two defects, but we consider here only the distance to $\mathrm{D}_{1 a}$ :

$$
\begin{aligned}
& \bmod \left[R\left(\mathrm{D}_{1 b}, \mathrm{D}_{1 a}\right), 2 a_{0}\right]=\bmod \left(7 a_{0}, 2 a_{0}\right)=1 a_{0}, \\
& \bmod \left[R\left(\mathrm{D}_{1 c}, \mathrm{D}_{1 a}\right), 2 a_{0}\right]=\bmod \left(8 a_{0}, 2 a_{0}\right)=0, \\
& \bmod \left[R\left(\mathrm{D}_{1 d}, \mathrm{D}_{1 a}\right), 2 a_{0}\right]=\bmod \left(15 a_{0}, 2 a_{0}\right)=1 a_{0}, \\
& \bmod \left[R\left(\mathrm{D}_{1 e}, \mathrm{D}_{1 a}\right), 2 a_{0}\right]=\bmod \left(14 a_{0}, 2 a_{0}\right)=0, \\
& \bmod \left[R\left(\mathrm{D}_{1 f}, \mathrm{D}_{1 a}\right), 2 a_{0}\right]=\bmod \left(15 a_{0}, 2 a_{0}\right)=1 a_{0} .
\end{aligned}
$$

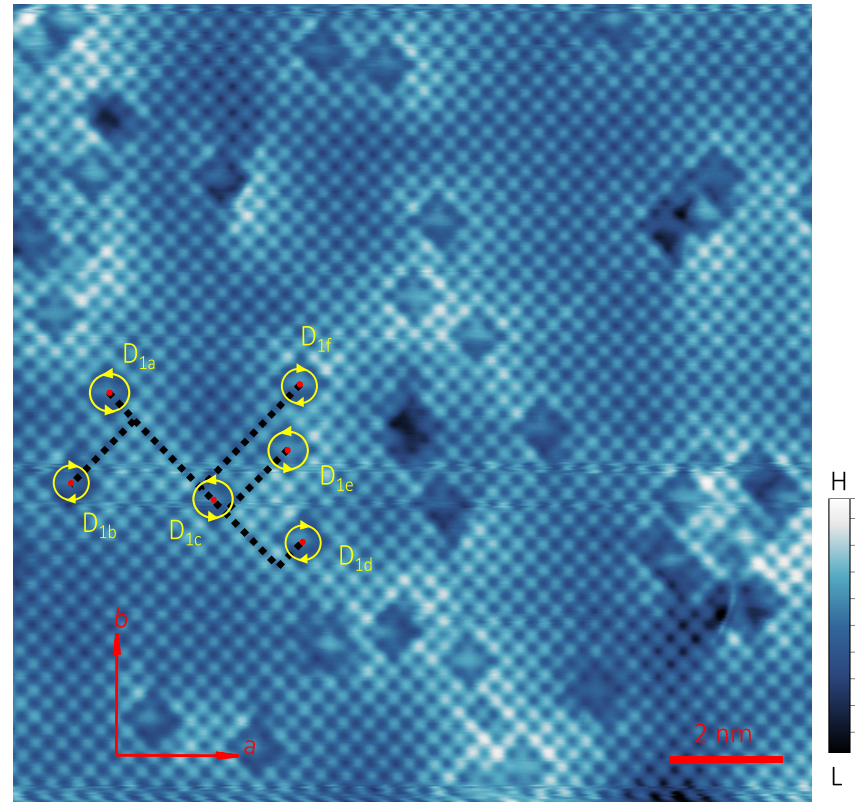

FIG. 8. The chiral behavior of the defect as a function of lattice site. The topography is the same as the one shown in Fig. 1(a). Yellow (solid) circles indicate the chirality of the corresponding $\mathrm{D}_{1}$-type defects.

From these numbers, we can expect that $\mathrm{D}_{1 c}$ and $\mathrm{D}_{1 e}$ have the same chirality as $\mathrm{D}_{1 a}$ and the remaining defects have the opposite chirality, as is also observed in Fig. 8.

\section{APPENDIX C: NUMERICAL SIMULATION}

\section{Simulation of the differential tunneling spectrum}

The LDOS calculation has been performed on the basis of a standard $t$-matrix approach where the momentum-integrated Green's function of the clean system is taken from Ref. [28].

We consider the one-particle Green's function $G_{0}(\mathbf{k}, E)$ as obtained from the self-consistent Born approximation plus a local potential scatterer with an assumed potential $V_{\mathbf{k}, \mathbf{k}^{\prime}}=V_{\mathrm{imp}}$ in momentum space. These parameters are used for a standard $t$-matrix approach to calculate the Fourier-transformed local density of states,

$$
\rho(\mathbf{q}, E)=\frac{1}{\pi} \sum_{\mathbf{k}} \operatorname{Im} G(\mathbf{k}, \mathbf{k}-\mathbf{q}, E),
$$

which is the quantity that can be directly measured by momentum-resolved STM/STS experiments. $G\left(\mathbf{k}, \mathbf{k}^{\prime}, E\right)$ is the retarded Green's function in the presence of one single impurity and is related to the retarded Green's function $G_{0}(\mathbf{k}, E)$ of the bulk material via the equation

$$
G\left(\mathbf{k}, \mathbf{k}^{\prime}, E\right)=G_{0}(\mathbf{k}, E)+G_{0}(\mathbf{k}, E) T_{\mathbf{k}, \mathbf{k}^{\prime}}(E) G_{0}\left(\mathbf{k}^{\prime}, E\right),
$$

where the energy-dependent $t$-matrix $T_{\mathbf{k}, \mathbf{k}^{\prime}}(E)$ is determined by the following self-consistency equation,

$$
T_{\mathbf{k}, \mathbf{k}^{\prime}}(E)=V_{\mathbf{k}, \mathbf{k}^{\prime}}+\sum_{\mathbf{k}^{\prime \prime}} V_{\mathbf{k}, \mathbf{k}^{\prime \prime}} G_{0}\left(\mathbf{k}^{\prime \prime}, E\right) T_{\mathbf{k}^{\prime \prime}, \mathbf{k}^{\prime}}(E) .
$$




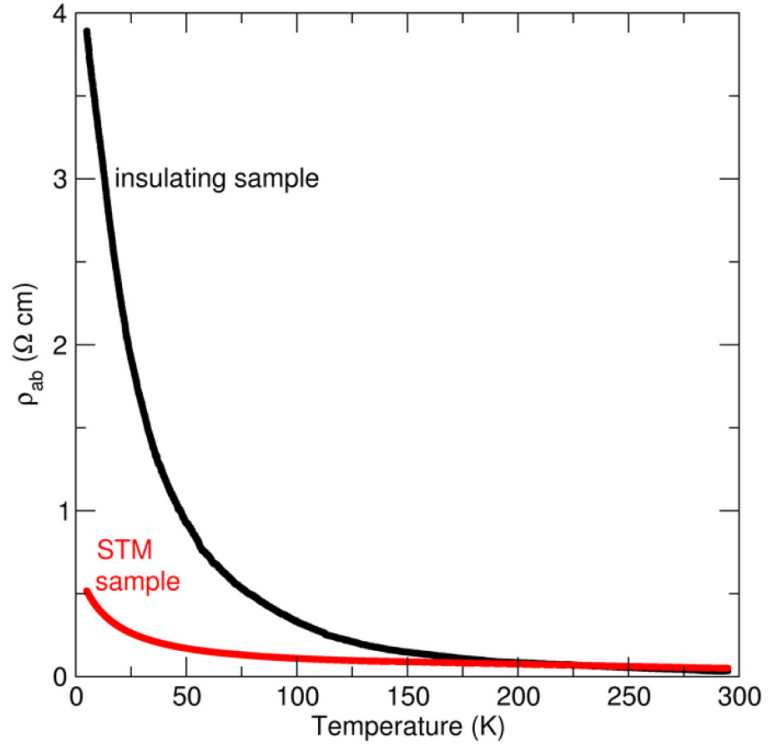

FIG. 9. The bulk resistivity plot as function of temperature. The bulk resistivity is plot for the sample used in the STM measurements (red, lower) and a highly insulating sample (black, upper).

Using the two functions $G_{0}(\mathbf{k}, E)$ and $V_{\mathbf{k}, \mathbf{k}^{\prime}}$ as input parameters we have solved Eq. (C3) self-consistently. The obtained result for $T_{\mathbf{k}, \mathbf{k}^{\prime}}(E)$ is inserted in Eq. (C2) for the full Green's function $G\left(\mathbf{k}, \mathbf{k}^{\prime}, E\right)$. Finally, this result is used in Eq. (C1) to evaluate the momentum-dependent variation of the density of states $\rho(\mathbf{q}, E)$ due to the presence of the impurity.

The corresponding impurity-induced variation of the density of states in real space is given by the Fourier transform of Eq. (C1),

$$
\rho\left(\mathbf{R}_{i}, E\right)=\frac{1}{N} \sum_{\mathbf{q}} \rho(\mathbf{q}, E) e^{i \mathbf{q} \mathbf{R}_{i}},
$$

where $\mathbf{R}_{i}$ is a lattice vector and $N$ is the total number of lattice sites in the system. Note that this quantity is proportional to the experimentally probed differential conductance $d I / d U$. The result for $\rho\left(\mathbf{R}_{i}=0, E\right)$ as a function of $E=U$ is shown in Fig. 3 (yellow line). Here, the specific location of the impu- rity, i.e., $\mathbf{R}_{i}=0$ is considered. For comparison, the calculated spectrum without impurity is also shown (green line).

The value of impurity potential $V_{\text {imp }}(28 \mathrm{meV})$ taken in the calculations is justified by $a b$ initio calculations in strongly correlated systems. Such calculations were done for example for the cuprates where the effective impurity potential for single oxygen vacancies was studied as a function of the distance to the impurity [29].

\section{Bond percolation analysis}

The numerical simulation of the bond percolation $[30,31]$ was performed on a $2 \mathrm{D}$ square lattice of $100 \times 100$ lattice sites according to the following procedure. At first we fixed the total number of defects to some chosen value and generated in total 1000 random distributions of these defects. For each of these distributions we counted the number of unbroken paths of connected defects which link at least two opposite boundaries of the lattice. If we found at least one of such paths for each single distribution we assigned the corresponding doping level metallic character.

Two defects were defined as connected (bond) if charge transfer is possible between these defects via the emergence of at least one bound state which is lower in energy than the states of a single defect. Experimentally, this situation appears in Fig. 4(d) where the Mott gap is partially closed between two distinct defects. From these considerations we fixed for our percolation simulation the size of a bond to four lattice constants.

The above steps were repeated for different doping levels and for each doping value we assigned either insulating or metallic character according to the criterion defined above. We found metallic character above a percolation threshold of $3.7 \%$ defect concentration.

\section{APPENDIX D: BULK RESISTIVITY}

A standard four-probe technique (5-300 K) was used in the as-grown $\mathrm{Sr}_{2} \mathrm{IrO}_{4}$ single crystals to measure the in-plane resistance to control the insulating behavior of the samples. Figure 9 shows representative resistivity data for different samples. The resistivity shows a semiconductorlike temperature dependence, which proves that the sample is still close to the Mott insulator regime.
[1] T. Hanaguri, C. Lupien, Y. Kohsaka, D.-H. Lee, M. Azuma, M. Takano, H. Takagi, and J. C. Davis, A 'checkerboard' electronic crystal state in lightly hole-doped $\mathrm{Ca}_{2-\mathrm{x}} \mathrm{Na}_{\mathrm{x}} \mathrm{CuO}_{2} \mathrm{Cl}_{2}$, Nature (London) 430, 1001 (2004).

[2] Y. Kohsaka, C. Taylor, K. Fujita, A. Schmidt, C. Lupien, T. Hanaguri, M. Azuma, M. Takano, H. Eisaki, H. Takagi, S. Uchida, and J. C. Davis, An intrinsic bond-centered electronic glass with unidirectional domains in underdoped cuprates, Science 315, 1380 (2007).

[3] E. H. da Silva Neto, P. Aynajian, A. Frano, R. Comin, E. Schierle, E. Weschke, A. Gyenis, J. Wen, J. Schneeloch, Z. $\mathrm{Xu}, \mathrm{S}$. Ono, G. Gu, M. Le Tacon, and A. Yazdani, Ubiquitous interplay between charge ordering and high-temperature superconductivity in cuprates, Science 343, 393 (2014).

[4] Y. K. Kim, O. Krupin, J. Denlinger, A. Bostwick, E. Rotenberg, Q. Zhao, J. Mitchell, J. Allen, and B. Kim, Fermi arcs in a doped pseudospin-1/2 Heisenberg antiferromagnet, Science 345, 187 (2014).

[5] Y. Cao, Q. Wang, J. A. Waugh, T. J. Reber, H. Li, X. Zhou, S. Parham, S.-R. Park, N. C. Plumb, E. Rotenberg, A. Bostwick, J. D. Denlinger, T. Qi, M. A. Hermele, G. Cao, and D. S. Dessau, Hallmarks of the Mott-metal crossover in the holedoped pseudospin-1/2 Mott insulator $\mathrm{Sr}_{2} \mathrm{IrO}_{4}$, Nat. Commun. 7, 11367 (2016). 
[6] Y. Kim, N. Sung, J. Denlinger, and B. Kim, Observation of a d-wave gap in electron-doped $\mathrm{Sr}_{2} \mathrm{IrO}_{4}$, Nat. Phys. 12, 37 (2016).

[7] G. Cao, J. Bolivar, S. McCall, J. E. Crow, and R. P. Guertin, Weak ferromagnetism, metal-to-nonmetal transition, and negative differential resistivity in single-crystal $\mathrm{Sr}_{2} \mathrm{IrO}_{4}$, Phys. Rev. B 57, R11039 (1998).

[8] B. J. Kim, H. Ohsumi, T. Komesu, S. Sakai, T. Morita, H. Takagi, and T. Arima, Phase-sensitive observation of a spin-orbital Mott state in $\mathrm{Sr}_{2} \mathrm{IrO}_{4}$, Science 323, 1329 (2009).

[9] J. Kim, D. Casa, M. H. Upton, T. Gog, Y.-J. Kim, J. F. Mitchell, M. van Veenendaal, M. Daghofer, J. van den Brink, G. Khaliullin, and B. J. Kim, Magnetic Excitation Spectra of $\mathrm{Sr}_{2} \mathrm{IrO}_{4}$ Probed by Resonant Inelastic X-Ray Scattering: Establishing Links to Cuprate Superconductors, Phys. Rev. Lett. 108, 177003 (2012).

[10] F. Wang and T. Senthil, Twisted Hubbard Model for $\mathrm{Sr}_{2} \mathrm{IrO}_{4}$ : Magnetism and Possible High Temperature Superconductivity, Phys. Rev. Lett. 106, 136402 (2011).

[11] Y. J. Yan, M. Q. Ren, H. C. Xu, B. P. Xie, R. Tao, H. Y. Choi, N. Lee, Y. J. Choi, T. Zhang, and D. L. Feng, Electron-Doped $\mathrm{Sr}_{2} \mathrm{IrO}_{4}$ : An Analogue of Hole-Doped Cuprate Superconductors Demonstrated by Scanning Tunneling Microscopy, Phys. Rev. X 5, 041018 (2015).

[12] I. Battisti, K. M. Bastiaans, V. Fedoseev, A. de la Torre, N. Iliopoulos, A. Tamai, E. C. Hunter, R. S. Perry, J. Zaanen, F. Baumberger, and M. P. Allan, Universality of pseudogap and emergent order in lightly doped Mott insulators, Nat. Phys. 13, 21 (2017).

[13] A. Chernyshev and R. Wood, Spin polarons and high- $T_{c}$ superconductivity, in Models and Methods of High- $T_{c}$ Superconductivity: Some Frontal Aspects, edited by J. K. Srivastava and S. M. Rao (Nova Science Publishers, Hauppauge, NY, 2003), Chap. 11 .

[14] J. M. Guevara, Z. Sun, E. M. Pärschke, S. Sykora, K. Manna, J. Schoop, A. Maljuk, S. Wurmehl, J. van den Brink, B. Büchner, and C. Hess, Spin-polaron ladder spectrum of the spin-orbitinduced Mott insulator $\mathrm{Sr}_{2} \mathrm{IrO}_{4}$ probed by scanning tunneling spectroscopy, Phys. Rev. B 99, 121114(R) (2019).

[15] T. F. Qi, O. B. Korneta, L. Li, K. Butrouna, V. S. Cao, X. Wan, P. Schlottmann, R. K. Kaul, and G. Cao, Spin-orbit tuned metal-insulator transitions in single-crystal $\mathrm{Sr}_{2} \mathrm{Ir}_{1-\mathrm{x}} \mathrm{Rh}_{\mathrm{x}} \mathrm{O}_{4}(0 \leqslant$ $x \leqslant 1$ ), Phys. Rev. B 86, 125105 (2012).

[16] X. Chen, T. Hogan, D. Walkup, W. Zhou, M. Pokharel, M. Yao, W. Tian, T. Z. Ward, Y. Zhao, D. Parshall, C. Opeil, J. W. Lynn, V. Madhavan, and S. D. Wilson, Influence of electron doping on the ground state of $\left(\mathrm{Sr}_{1-\mathrm{x}} \mathrm{La}_{\mathrm{x}}\right)_{2} \mathrm{IrO}_{4}$, Phys. Rev. B 92, 075125 (2015).

[17] A. de la Torre, S. McKeown Walker, F. Y. Bruno, S. Riccó, Z. Wang, I. Gutierrez Lezama, G. Scheerer, G. Giriat, D. Jaccard, C. Berthod, T. K. Kim, M. Hoesch, E. C. Hunter, R. S. Perry, A. Tamai, and F. Baumberger, Collapse of the Mott Gap and
Emergence of a Nodal Liquid in Lightly Doped $\mathrm{Sr}_{2} \mathrm{IrO}_{4}$, Phys. Rev. Lett. 115, 176402 (2015).

[18] O. B. Korneta, T. Qi, S. Chikara, S. Parkin, L. E. De Long, P. Schlottmann, and G. Cao, Electron-doped $\mathrm{Sr}_{2} \mathrm{IrO}_{4-\delta}(0 \leqslant \delta \leqslant 0.04)$ : Evolution of a disordered $J_{\text {eff }}=1 / 2$ Mott insulator into an exotic metallic state, Phys. Rev. B 82, 115117 (2010).

[19] F. Mott, Metal-Insulator Transitions (Taylor and Francis, London, 1990).

[20] B. Keimer, S. A. Kivelson, M. R. Norman, S. Uchida, and J. Zaanen, From quantum matter to high-temperature superconductivity in copper oxides, Nature (London) 518, 179 (2015).

[21] K. Manna, G. Aslan-Cansever, A. Maljuk, S. Wurmehl, S. Seiro, and B. Büchner, Flux growth of $\mathrm{Sr}_{\mathrm{n}+1} \mathrm{Ir}_{\mathrm{n}} \mathrm{O}_{3 \mathrm{n}+1}(\mathrm{n}=1,2, \infty)$ crystals, J. Cryst. Growth 540, 125657 (2020).

[22] R. Schlegel, T. Hänke, D. Baumann, M. Kaiser, P. Nag, R. Voigtländer, D. Lindackers, B. Büchner, and C. Hess, Design and properties of a cryogenic dip-stick scanning tunneling microscope with capacitive coarse approach control, Rev. Sci. Instrum. 85, 013706 (2014).

[23] Q. Huang, J. Soubeyroux, O. Chmaissem, I. N. Sora, A Santoro, R. Cava, J. Krajewski, and W. Peck, Jr., Neutron powder diffraction study of the crystal structures of $\mathrm{Sr}_{2} \mathrm{RuO}_{4}$ and $\mathrm{Sr}_{2} \mathrm{IrO}_{4}$ at room temperature and at $10 \mathrm{~K}$, J. Solid State Chem. 112, 355 (1994).

[24] Y. Okada, D. Walkup, H. Lin, C. Dhital, T.-R. Chang, S. Khadka, W. Zhou, H.-T. Jeng, M. Paranjape, A. Bansil, Z. Wang, S. D. Wilson, and V. Madhavan, Imaging the evolution of metallic states in a correlated iridate, Nat. Mater. 12, 707 (2013).

[25] J. Dai, E. Calleja, G. Cao, and K. McElroy, Local density of states study of a spin-orbit-coupling induced Mott insulator $\mathrm{Sr}_{2} \mathrm{IrO}_{4}$, Phys. Rev. B 90, 041102(R) (2014).

[26] H. Zhao, S. Manna, Z. Porter, X. Chen, A. Uzdejczyk, J. Moodera, Z. Wang, S. D. Wilson, and I. Zeljkovic, Atomicscale fragmentation and collapse of antiferromagnetic order in a doped Mott insulator, Nat. Phys. 15, 1267 (2019).

[27] Z. Wang, D. Walkup, Y. Maximenko, W. Zhou, T. Hogan, Z. Wang, S. D. Wilson, and V. Madvahan, Doping induced Mott collapse and possible density wave instabilities in $\left(\mathrm{Sr}_{1-\mathrm{x}} \mathrm{La}_{\mathrm{x}}\right)_{3} \mathrm{Ir}_{2} \mathrm{O}_{7}$, npj Quantum Mater. 4, 43 (2019).

[28] E. M. Pärschke, K. Wohlfeld, K. Foyevtsova, and J. van den Brink, Correlation induced electron-hole asymmetry in quasitwo-dimensional iridates, Nat. Commun. 8, 686 (2017).

[29] L.-L. Wang, P. J. Hirschfeld, and H.-P. Cheng, Ab initio calculation of impurity effects in copper oxide materials, Phys. Rev. B 72, 224516 (2005).

[30] M. E. J. Newman and R. M. Ziff, Efficient Monte Carlo Algorithm and High-Precision Results for Percolation, Phys. Rev. Lett. 85, 4104 (2000).

[31] M. F. Sykes and J. W. Essam, Critical percolation probabilities by series methods, Phys. Rev. 133, A310 (1964). 